EPJ Web of Conferences 19, 03004 (2012)

DOI: $10.1051 /$ epjconf/20121903004

(C) Owned by the authors, published by EDP Sciences, 2012

\title{
Stellar population gradients in isolated, local group dwarf galaxies
}

\author{
S.L. Hidalgo ${ }^{a}$, for the LCID team \\ Instituto de Astrofísica de Canarias, 38200 La Laguna, Spain
}

\begin{abstract}
We discuss the detailed star formation as a function of radius that we have derived for the LCID galaxies, with particular emphasis on the stellar populations gradient and the effect of the UV-background.
\end{abstract}

\section{INTRODUCTION}

Among the mechanisms proposed to shape the evolution of dwarf galaxies, SN feedback, SN heating, UV-background, and self-shielding may have a dependency on the galactocentric radius [3, 9, 11, 14]. If this is the case, they may have affected the spatial distribution of the star formation. The study of the stellar populations distribution should provide hints on the role played by these mechanisms and how they shaped the galaxy evolution in a cosmological context.

The results presented in this paper are based on the observation of the LCID project (Local Cosmology form the Isolated Dwarfs). The aim of this project is to recover the full SFHs of six isolated dwarf galaxies of the Local Group: Phoenix, LGS-3, Cetus, Tucana, IC1613, and Leo-A, which have been already described in [4-7, 10], and [2]. We have selected a subsample of the dwarf galaxies of the LCID project to obtain the SFH as a function of radius: two transition galaxies (Phoenix and LGS-3) and two dSph galaxies (Cetus and Tucana).

\section{STAR FORMATION HISTORY AS A FUNCTION OF RADIUS}

Figure 1 shows the color-magnitude diagrams (CMDs) of Cetus, Tucana, LGS-3, and Phoenix of four regions defined as a function of the scale length of each galaxy. The CMDs of each galaxy, except those of Phoenix, contain the same number of stars. It is interesting to note that there is no a clear change with radius of the blue-plume of stars of Cetus and Tucana. This stars have been identify as a blue straggler star population by [8]. However, in the case of LGS-3 and Phoenix, there is a clear change in the main-sequence of young stars being less populated toward the outer regions. Finally, all the CMDs of the galaxies looks very similar beyond two scale lengths.

Using the CMDs of Fig. 1 we have obtained the SFHs as a function of galactocentric radius, which is shown in Fig. 2. The two dSphs, Cetus and Tucana, show a small age gradient with radius. Cetus shows a star formation ending at $\sim 9 \mathrm{Gyr}$ at the center but slightly shifted to older ages at outer radius. In Tucana, the end of the star formation is centered around $7 \mathrm{Gyr}$ in the innermost region but it seems to end around $9 \mathrm{Gyr}$ at the outermost regions of the galaxy. On the other hand, the two transition galaxies LGS-3 and Phoenix show a smooth age gradient toward older ages with increasing radius. This is clear in LGS-3 with a decreasing relative contribution of young and intermediate age stars respect to older

\footnotetext{
ae-mail: shidalgo@iac.es
}

This is an Open Access article distributed under the terms of the Creative Commons Attribution-Noncommercial License 3.0, which permits unrestricted use, distribution, and reproduction in any noncommercial medium, provided the original work is properly cited. 


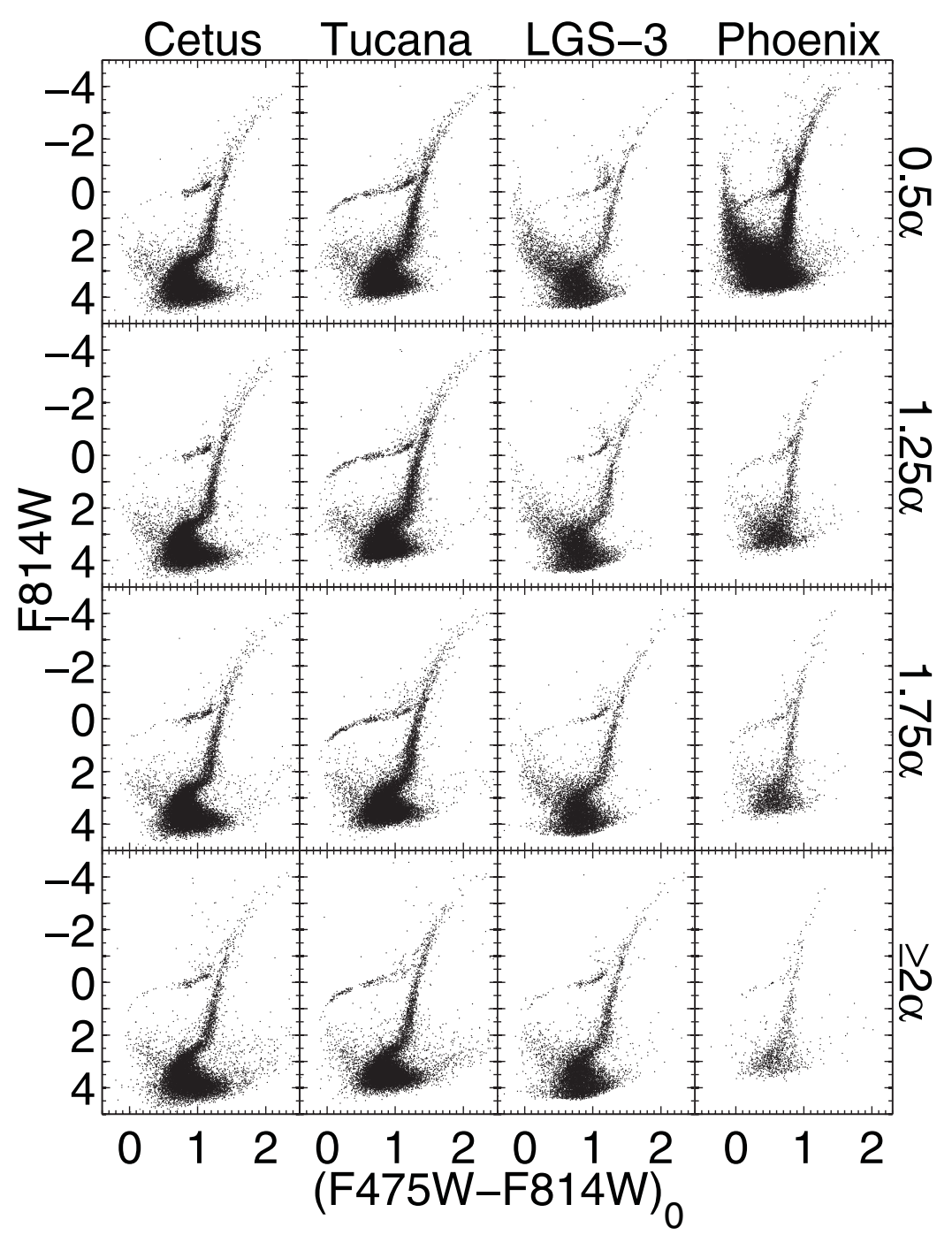

Figure 1. Color-magnitude diagrams of the galaxies as a function of the distance to the center. Each row corresponds to a region in which the observed fields were divided. The mean distance to the center is marked in scale length units on the right side of the figure.

stars at increasing radii. However, stars older than $\sim 8$ Gyr are located at all radii. In the case of Phoenix, there is also a gradient toward older ages with increasing radius, although beyond two scale lengths from the center the mean age of the stars increase.

\section{THE EFFECT OF THE UV-BACKGROUND IN THE SFHS OF ISOLATED DWARFS}

The UV-background acts not only by suppressing the gas accretion in the lowest mass galaxies but also by heating the remaining gas above the Virial temperature of the DM halo and expelling it [1]. Thermal feedback may be necessary prior to the UV-background to shut down the star formation [12] and [9]. In any case, the final result is that for the lowest mass galaxies, the star formation should switched off at redshift $z \sim 6$ in a very short time in the whole galaxy. As a result, lower mass galaxies will only show 


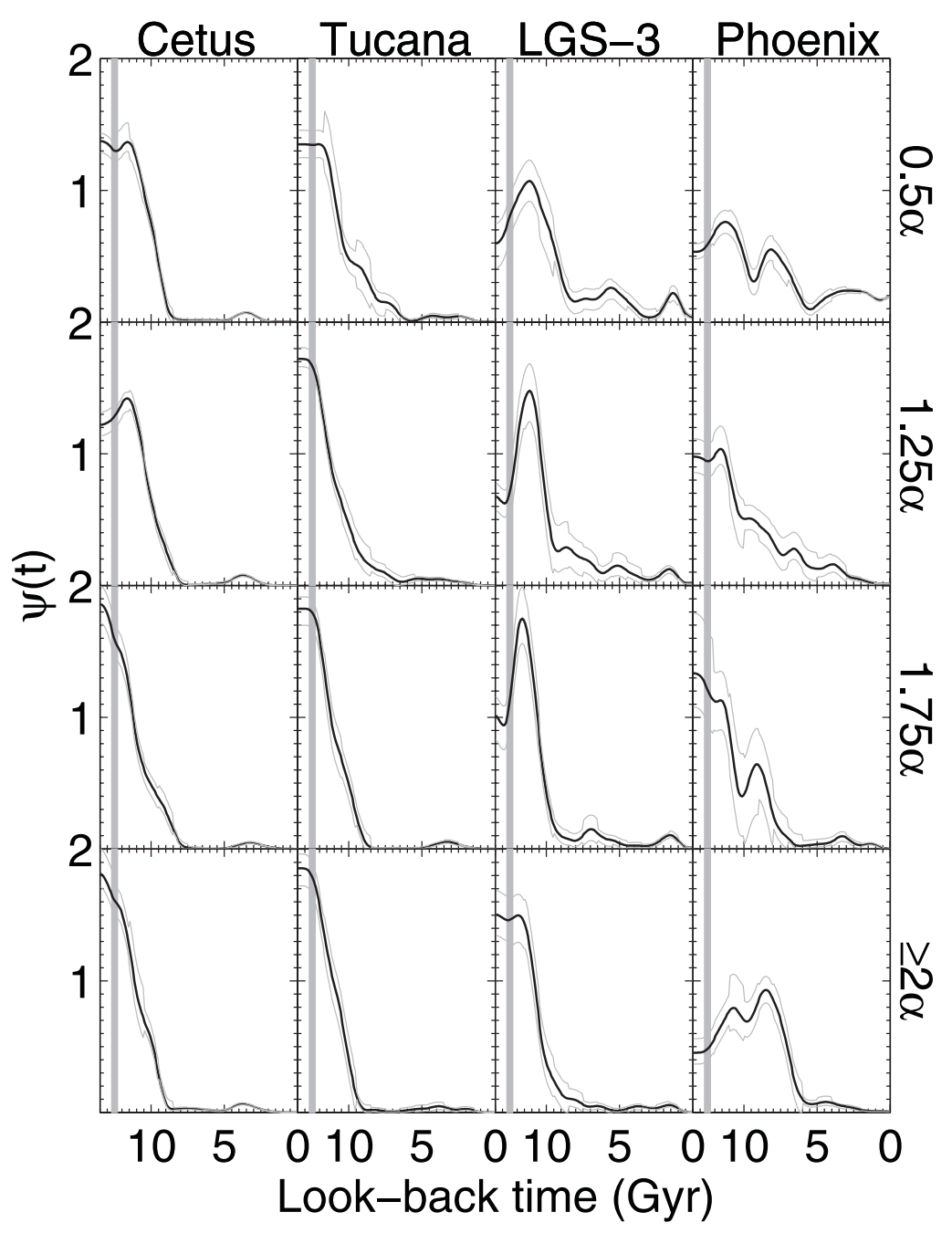

Figure 2. SFHs as a function of radius obtained from the CMDs shown in 1 . The gray shaded region points to the epoch of reionization.

a short formation time interval. However, in higher mass galaxies which are able to keep dense gas in the center, the self-shielding prevents that UV-background stops the star formation [9, 13], allowing a longer formation time interval.

To study the effect of the UV-background on the SFH as a function of radius we have marked the epoch of reionization (EoR) on 2. The EoR can be placed between redshift 6 to 12 []. At first glance, it seems that all galaxies have formed stars after the EoR at all radii. In fact, the main peak of star formation in the transition galaxies is located after the EoR. Although the star formation in Cetus and Tucana end earlier at outer radius, it seems that some star formation is needed after the EoR. However, we know that observational effects tend to broaden the main features of the SFH [4]. Except for Phoenix in which there are high star formation after the EoR even at a distance beyond two scale lengths, the observational effects could misinterpret the results for the other galaxies, especially at outer radius where the end of the star formation is closer to the EoR. 


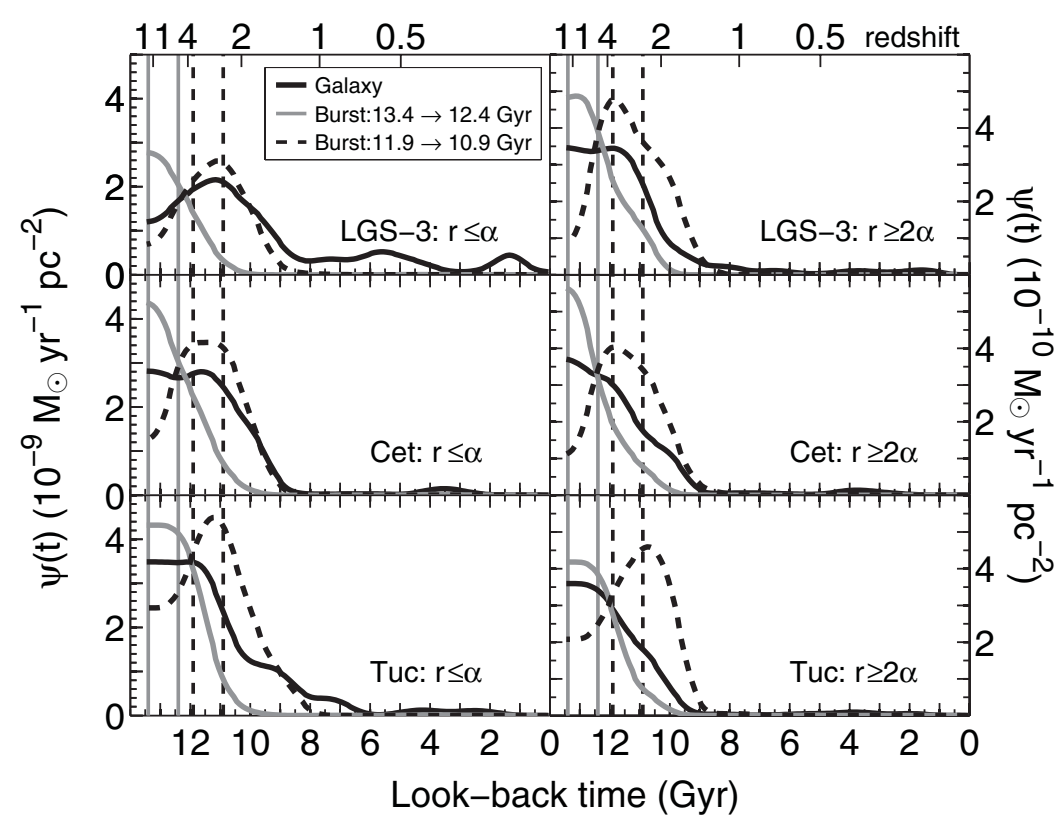

Figure 3. The effect of UV-background in the SFH of two regions $(r \leq \alpha$ and $r \geq 2 \alpha)$ of LGS-3, Cetus, and Tucana. Two input mock star bursts and their recovered SFHs located before (gray, solid line) and after (black, dashed line) the EoR are shown. The resolved SFH of each galaxy is shown with a solid, black line.

We have preformed several SFH mock tests to elucidate whether the SFHs hold a clear, true signature of the EoR. We show the result of two mock SFHs in Fig. 3: one for a burst of stars formed before the EoR between 13.4 and 12.4 Gyr ago, and another for stars formed after the EoR between 11.9 and $10.9 \mathrm{Gyr}$ ago. We have preformed this analysis at two distances from the center $(r \leq \alpha$ and $r \geq 2 \alpha)$ for those galaxies in which the results are not completely clear. The results show that for $r \leq \alpha$, the model with all stars formed before the EoR is inconsistent with the SFHs of the galaxies. This is clearly shown for LGS-3 for which the model with all stars formed after the EoR is a good match for its SFH. For Cetus and Tucana, it seems that the best match is produced by a mixture of stars formed before and after the EoR. For stars with $r \geq 2 \alpha$, it is clear that neither model by itself is able to reproduce the SFHs. In this case, a mixture of both models with star formation before and after the EoR is required in order to reproduce the SFHs. In the case of Tucana, the model with all the star formed before the EoR is even in better agreement. With this result, we can say tentatively that The UV-background did not stop the star formation at $r \leq \alpha$ and, hence, the galaxies were self-shielded at this radius. At $r \geq 2 \alpha$, the galaxies still show evidences of self-shielding against the UV-background although the relative contribution of star formation before the EoR is larger.

\section{CONCLUSIONS}

The SFH of the transition galaxies of the LCID project show a stellar population gradient with the mean age of the stars being older at increasing radius. There is no clear evidences of the UV-background ending the star formation in the LCID galaxies at any radius almost up to two scale lengths.

\section{References}

[1] Bullock, J. S., Kravtsov, A. V., \& Weinberg, D. H., ApJ, 539, (2000) 517

[2] Cole, A. A., et al., ApJL, 659, (2007) L17 
[3] Ferrara, A., \& Tolstoy, E., MNRAS, 313, (2000) 291

[4] Hidalgo, S. L., et al., ApJ, 730, (2011) 14

[5] Hidalgo, S. L., Aparicio, A., Martínez-Delgado, D., \& Gallart, C., ApJ, 705, (2009) 704

[6] Monelli, M., et al., ApJ, 720, (2010) 1225

[7] Monelli, M., et al., ApJ, 722, (2010) 1864

[8] Monelli, M., Cassisi, S., Mapelli, M., et al., arXiv, 1109.6875, (2011)

[9] Sawala, T., Scannapieco, C., Maio, U., \& White, S., MNRAS, 402, (2010) 1599

[10] Skillman et al. (in preparation)

[11] Stinson, G. S., Dalcanton, J. J., Quinn, T., et al., MNRAS, 395, (2009) 1455

[12] Susa, H., \& Umemura, M., ApJL, 610, (2004) L5

[13] Tajiri, Y., \& Umemura, M., ApJ, 502, (1998) 59

[14] Tassis, K., Abel, T., Bryan, G. L., \& Norman, M. L., ApJ, 587, (2003) 13 